\section{Riociguat ist bei PAH und CTEPH langfristig effektiv}

Halank M et al. Riociguat for pulmonary arterial hypertension and chronic thromboembolic pulmonary hypertension: Results from a phase II long-term extension study. Respir Med 2017; 128: 50 - 56. doi:10.1016/j.rmed.2017.05.008

Riociguat stimuliert die lösliche Guanylatzyklase, sensibilisiert für endogenes NO und erhöht das cGMP. Bei Patienten mit einer pulmonal-arteriellen Hypertonie (PAH) und chronischen thromboembolischen pulmonalen Hypertonie (CTEPH) verlängerten 12 Wochen Riociguat die 6-Minuten-Gehstrecke, steigerten die körperliche Belastbarkeit insgesamt und verbesserten die hämodynamischen Parameter. Halank et al. stellen nun die Behandlungsergebnisse nach 77 Monaten Riociguat vor.

Riociguat ist für die PAH und die inoperable oder rezidivierende CTEPH zugelassen. Die gesteigerte Produktion des Second Messenger cGMP bewirkt eine Vasodilatation und hat antiinflammatorische, antiproliferative und antifibrotische Effekte. Aufnahmevoraussetzungen der Studie waren eine PAH oder inoperable CTEPH (WHO-Funktionsklasse II/III, mittlerer pulmonal-arterieller Wider- stand $>300$ dyn $\mathrm{s} \cdot \mathrm{cm}^{-5}$, mittlerer pulmonal-arterieller Druck $\geq 25 \mathrm{mmHg}$ ) und ein abgeschlossener 1. Untersuchungsteil von 12 Wochen. Die nicht kontrollierte Open-Label-Langzeitstudie (LTE) fand an 13 deutschen Zentren statt.

Insgesamt 68 Patienten nahmen maximal 2,5 mg tid Riociguat ein. In 41 Fällen bestand eine inoperable/rezidivierte CTEPH und in 27 Fällen eine PAH. 6 Patienten ( $9 \%$ ) erhielten zusätzlich Bosentan. Nach durchschnittlich 77 Monaten nahmen 53\% der Patienten weiterhin Riociguat ein. 47\% hatten die Behandlung abgebrochen. Die Riociguatdosis betrug bei $74 \%$ weiterhin $2,5 \mathrm{mg}$ tid. Während der LTE erhielten 30 Patienten (44\%) zusätzlich Endothelinrezeptor-Antagonisten und/oder Prostanoide. $32 \mathrm{~Pa}$ tienten (47\%) führten die Monotherapie mit Riociguat fort. Fast alle Patienten berichteten über Nebenwirkungen (93\%), die bei PAH und CTEPH nicht unterschiedlich häufig vorkamen. Führend waren beherrschbare Nasopharyngitiden (57\%) und periphere Ödeme (37\%). Von schweren Komplikationen war die CTEPH-Gruppe öfter betroffen. 3 Synkopen und 2 Verschlechterungen des pulmonalen Hochdrucks wurden auf Riociguat zurückgeführt. 3 Patienten mit PAH bekamen Hämoptysen, die nicht mit der Einnahme assoziiert waren. 9\% der Patienten brachen die Riociguattherapie wegen Nebenwirkungen ab.
Die funktionellen Ergebnisse nach 48 Monaten bestätigten den langfristigen Nutzen von Riociguat $(n=44)$.

95\% der Fälle wiesen weiterhin eine verbesserte oder stabilisierte WHO-Funktionsklasse auf (PAH 100\% und CTEPH $92 \%)$. In 6 Jahren starben $12 \%$ der $\mathrm{Pa}$ tienten an einer Krankheitsprogression. Nach 9, 36 und 72 Monaten hatten 73\%, 49\% und 35\% nicht progrediente Krankheitsverläufe. Die Überlebensraten betrugen $97 \%$, $91 \%$ und $81 \%$.

FAZIT

Nach einer Behandlungszeit von 4 Jahren bestätigte sich die Riociguatwirksamkeit mit einer anhaltend verbesserten Gehstrecke und Gesamtbelastbarkeit sowie einem vertretbaren Nebenwirkungsprofil, so die Autoren. Die Überlebensdaten nach 6 Jahren reflektieren die Effektivität von Riociguat. Eine klinische Verschlechterung war als Beginn einer zusätzlichen Medikation definiert. Daraus resultierten laut den Autoren die vergleichsweise hohen Progressionsraten.

Dr. med. Susanne Krome, Melle 